\title{
A note on modeling a void generation and contraction in complex plasma
}

\author{
Abstract \\ An empty region (void) has been observed in many experiments on complex (dusty) \\ plasma in particular which were taken place at the International Space Station. Here \\ the known model of Avinash, Bhattacharjee and Hu of formation of void in complex \\ plasma is considered to provide simulations of voids evolution from initially \\ unstable flow mode. The model has been transformed into the divergent form. The \\ MUSCL scheme of the second approximation order in space and in time has been \\ applied for the hydrodynamic part of the model and a MINMOD limiter has been \\ used for obtaining monotonic solution. As a result the dynamics of void's growth and \\ its partial contraction to the center have been obtained at the final stage of the void \\ evolution.
}

Keywords: dusty plasma, complex plasma void, numerical methods, MUSCL scheme, plasma simulation
Volume 2 Issue 5 - 2018

OV Kravchenko,' OA Azarova ${ }^{2}$

'Scientific and Technological Center of Unique Instrumentation of RAS, Russia

${ }^{2}$ Dorodnicyn Computing Centre, Federal Research Center,

"Computer Science and Control" of RAS, Russia

Correspondence: Olga A Azarova, Dorodnicyn Computing Centre, Federal Research Center, “Computer Science and Control" of RAS, Vavilova str. 40, Russia, Moscow, Tel +7499|350002, Email olgazarov@gmail.com

Received: August 24, 2018 | Published: September 26, 2018

\section{Nomenclature \\ $n_{d} n_{e} \quad=$ densities of dust and electrons components \\ $v_{d} v_{i} \quad=$ velocities of dust and ions components \\ $E \quad=$ electric field currency \\ $F_{d} \quad=$ ion-drag force \\ $D_{0} \quad=$ diffusion coefficient \\ $t, x, y \quad=$ time and spatial variables \\ $a, b \quad=$ fit parameters of ion-drag force approximation \\ $\mu \quad=$ coefficient of ions mobility \\ $\alpha_{0} \quad=$ friction coefficient \\ $\tau_{d^{\prime}} \tau_{i} \quad=$ coefficients of normalized temperature for dust} and ion species

$n_{i} \quad=$ amount of the grid points in the $x$-direction

Index “ " refers to the initial parameters

\section{Introduction}

Recently a field connected with the research of complex plasma is widely investigated. ${ }^{1-3}$ One of the first complex plasma patterns containing a domain which is free of dust particles (void) was observed in the course of the experiments on the board of the International Space Station ${ }^{3}$. Afterwards similar patterns were found at the laboratory conditions. ${ }^{4-6}$ To describe such a pattern formation the model of Avinash, Bhattacharjee and $\mathrm{Hu}$ was introduced. ${ }^{7,8}$ In this model the evolution of the dynamics of a single symmetric void from an equilibrium state is described by electro-hydrodynamic equations which take into account the effect of an ion attraction force as a nonlinear function of the speed of ions. The algorithm for modeling the appearance of complex plasma void by means of the model has been presented for the case of cylindrical geometry of the electrical field ${ }^{8}$. Also, generation of a single symmetric void and concentric symmetrical voids in unmoving flows of low-temperature complex plasma has been obtained. ${ }^{9,10}$ A study of generation of both voids in moving flows ${ }^{11}$ and unmoving media was presented. ${ }^{12}$ Recently the Lax's scheme with re-calculation ${ }^{11}$ and the complex conservative difference scheme ${ }^{13}$ have been used for the hydrodynamic part of the model. At the same time, the MUSCL scheme is a widely using tool in hydrodynamic ${ }^{14}$ and plasma simulations. ${ }^{15}$ This paper is devoted to the MUSCL scheme application to a void evolution with respect to the contraction process. The simulations are based on the algorithm with using a predictor-corrector procedure for obtaining the second approximation order in space and in time and a MINMOD limiter is used for obtaining monotonic solution. Presented results can be used in research of dusty plasma dynamics under the conditions of microgravity which may associate with the conditions in space.

\section{Model}

Modeling is based on the model Avinash et al. ${ }^{8}$ Governing equations considered in dimensionless form with respect to the normalizing parameters ${ }^{8}$ have been applied:

$$
\begin{gathered}
\frac{\partial v_{d}}{\partial t}+v_{d} \frac{\partial v_{d}}{\partial x}=F_{d}-E-\alpha_{0} v_{d}-\frac{\tau_{d}}{n_{d}} \frac{\partial n_{d}}{\partial x}, \\
\frac{\partial n_{d}}{\partial t}=-\frac{\partial\left(n_{d} v_{d}\right)}{\partial x}+D_{0} \frac{\partial^{2} n_{d}}{\partial x^{2}}, \\
\frac{d n_{e}}{d x}=-\frac{n_{e} E}{\tau_{i}}, \\
\frac{d E}{d x}=1-n_{e}-n_{d}, \\
F_{d}=\frac{a E}{b+\left|v_{i}\right|^{3}}, \quad v_{i}=\mu E .
\end{gathered}
$$

Here concentrations of dust and electrons components are $n_{d^{\prime}} n_{e}$; 
velocities of dust and ions components are $v_{d} v_{i}$; electric field currency is $E$, ion-drag force is $F_{d}$, diffusion coefficient is $D_{0}$. In the system (1)(5): (1) is the dust momentum and (2) is the dust continuity equations, (3) is the balance equation of electrons neglecting the electron inertia, the Poisson law which completes the nonlinear system of equations, and the expressions for ion-drag force presented in $(4,5)$ constitute the governing equations. Also, one can consider equations (1) and (2) as the hydrodynamic part of the model ${ }^{9-12}$ and the equations (3)-(5) are the electrostatic part of it.

\section{Methods}

The hydrodynamic part of the model can be expressed in the divergent form

$$
\frac{\partial \mathbf{u}}{\partial t}+\frac{\partial \mathbf{F}}{\partial x}=\mathbf{f}
$$

where $\mathbf{u}=\left(\begin{array}{l}n_{d} \\ v_{d}\end{array}\right), \mathbf{F}=\left(\begin{array}{c}n_{d} v_{d} \\ 0.5 v_{d}^{2}+\tau_{d} \ln n_{d}\end{array}\right), \mathbf{f}=\left(\begin{array}{c}D_{0} \frac{\partial^{2} n_{d}}{\partial x^{2}} \\ F_{d}-E-\alpha_{0} v_{d}\end{array}\right)$.

Earlier the centered scheme was applied to the hydrodynamic part of the model as well as the Lax's scheme with re-calculation. ${ }^{10}$ The eq. (6) was approximated with the second order using central scheme and the method of Runge-Kutta of the fourth order was used to calculate (3), (4). ${ }^{10}$ Also, in the numerical simulations the next restrictions were imposed: if $n_{d}>1$ then $n_{d}$ was applied by 1 and $v_{d}$ was applied by $v_{d 0}$. Here we apply the MUSCL scheme to (6). The algorithm is free of any restrictions on $n_{d}$ and $v_{d}$. A predictor-corrector procedure is used for obtaining the second approximation order in space and in time. To obtain monotonic shape of the numerical solution a MINMOD limiter
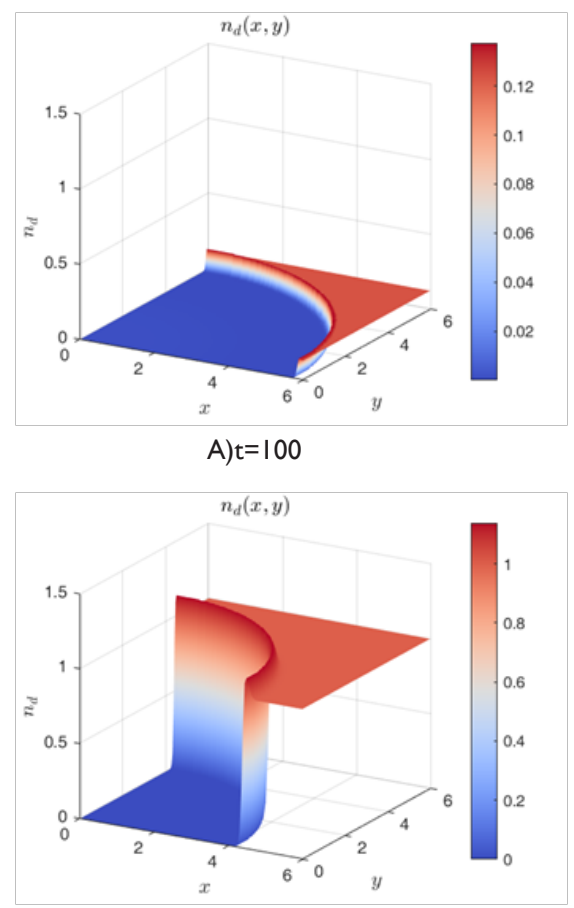

C) $\mathrm{t}=700$ based on the values $n_{d}$ is utilized. Parameters of the simulations are collected in Table 1 .

Table I Parameters of the simulations

\begin{tabular}{ll}
\hline Parameter & Value \\
\hline$D_{0}$ & 0 \\
$\tau_{i}$ & 0.125 \\
$\tau_{\mathrm{d}}$ & 0.001 \\
$a$ & 7.5 \\
$b$ & 1.6 \\
$\tau_{0}$ & 2 \\
$\mu$ & 1.5 \\
$n \mathrm{e}_{0}$ & 0.999 \\
$E_{0}$ & $10^{-6}$ \\
\hline
\end{tabular}

\section{Results}

The evolution of void generation in time in the initially unmoving flow $\left(v_{d 0}=0\right)$ is presented in Figure 1 and Figure 2. The dynamics of dusty particles density $n_{d}$ is shown in Figures $1 \mathrm{~A}-1 \mathrm{D}$ and the dynamics of dusty particles velocity $v_{d}$ is shown in Figures 2A-2D. Here twodimensional plots for $n_{d}, v_{d}$ obtained by the rotation technique are presented. The mechanism of generation of voids is based on the superposition of the electrical field and the ion attraction force action. Void develops in time from the uniformly distributed density and velocity values with constant parameters. The initial circular structure is shown in Figure 1A, the void becomes saturated at $t=300$ and the contraction process occurs up to $t=1000$.

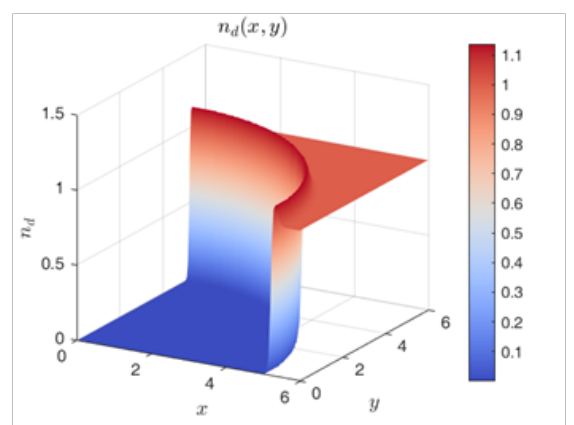

B) $\mathrm{t}=300$

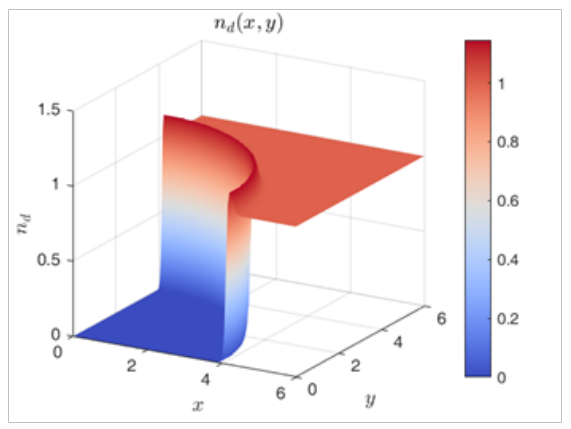

D) $\mathrm{t}=1000$

Figure I Evolution of the density of dust particles $n_{d}$. 


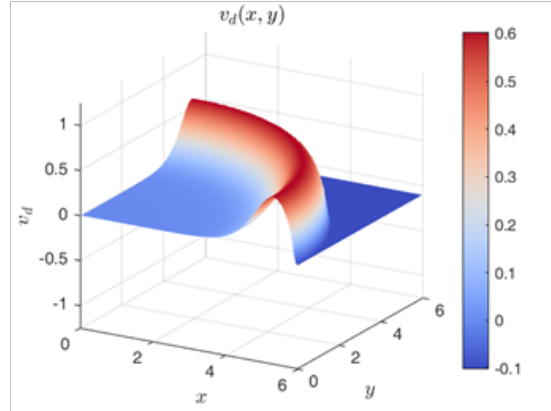

A) $\mathrm{t}=100$

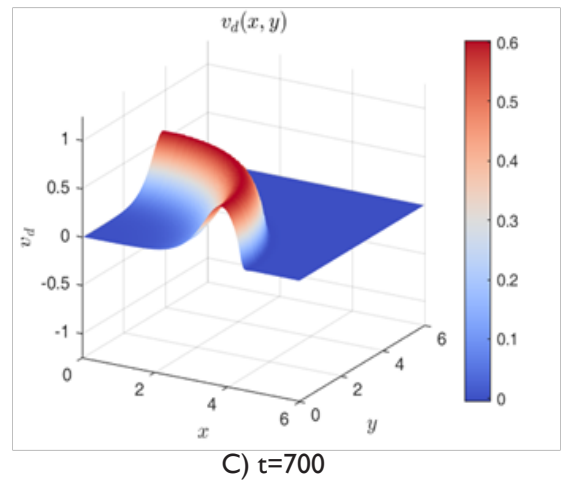

Figure 2 Evolution of the velocity of dust particles $v_{d}$.

\section{Conclusion}

Generation of a pattern with empty region (void) has been modelled numerically in complex plasma. The modeling is based on the Avinash, Bhattacharjee and Hu model of a void formation. The model has been reduced to the divergent form and the MUSCL scheme of the second approximation order has been used for the hydrodynamic part of the model. Dynamics of the void parameters such as density and velocity of dust particles has been obtained. It has been shown that after the initial stage of the circular void structure formation and before the steady state establishing the intermediate stage occurs of a void subsequent expansion and partial contraction to the center.

\section{Acknowledgements}

None.

\section{Conflict of interest}

The author declares that there is no conflict of interest.

\section{References}

1. Khrapak AG, Molotkov VI, Lipaev AM, et al. 2016. Complex Plasma Research under Microgravity Conditions: PK-3 Plus Laboratory on the International Space Station. Contributions to Plasma Physics. 2016;56(3-4):253-262.

2. Molotkov V, Thomas H, Lipaev V, et al. Complex (dusty) plasma research under microgravity conditions: PK-3 Plus Laboratory on the International Space Station. Int J Microgravity Sci Appl. 2015;35(3):18.

3. Fortov VE, Morgill GE. Complex and dusty plasmas: from laboratory to Space. New Jersey: CRC Press: 2009. 440 p.

4. Feng H, Mao-Fu Y, Long W, et al. Voids in experimental dusty plasma. Chin Phys Lett. 2004;21(1):121-124.

5. Sarkar S, Mondal M, Bose M, et al. Observation of external control and

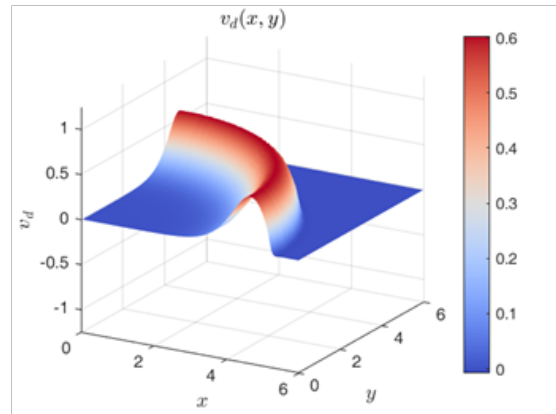

B) $t=300$

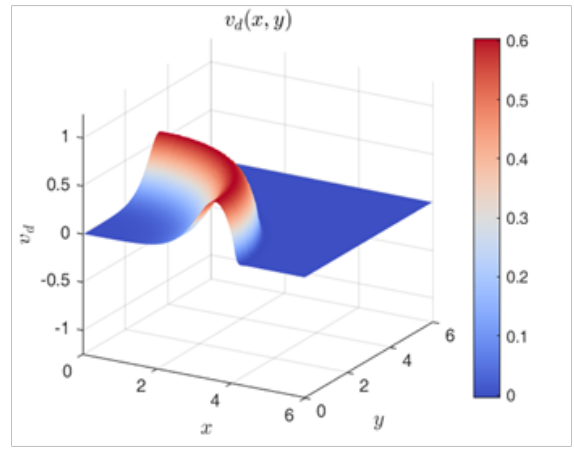

D) $\mathrm{t}=1000$

formation of a void in cogenerated dusty plasma. Plasma Sources Sci Technol. 2015;24(3):1-7.

6. Feng H, Maofu Y, Long W. Pattern phenomena in an RF discharge dusty plasma system. Science in China Series G: Physics, Mechanics \& Astronomy. 2006;49(5):588-596.

7. $\mathrm{Ng} \mathrm{CS}$, Bhattacharjee A, Hu S, et al. Generalizations of a nonlinear fluid model for void formation in dusty plasmas. Plasma Phys Control Fusion. 2007;49:1583-1597.

8. Avinash K, Bhattacharjee A, Hu S. Nonlinear theory of void formation in colloidal plasmas. Ph ys Rev Lett. 2003;90(7):075001.

9. Kravchenko OV, Pustovoit VI. Numerical simulation of dynamics of concentric dusty-plasma structures. Moscow: 15th International Workshop on Magneto-Plasma Aerodynamics; 2016. 8 p.

10. Kravchenko OV. Simulation of spatially localized dusty plasma structures in complex plasma. Nanostructures Mathematical physics and modeling. 2016;15(2):51-61 (in Russian).

11. Kravchenko OV, Azarova OA, Lapushkina TA. Dusty plasma void dynamics in unmoving and moving flows. Milan: 7th European Conference for Aeronautics and Space Sciences; 2017. 12 p.

12. Kravchenko OV, Azarova OA. Generation of voids in unmoving and moving dusty plasma. Nanostructures Mathematical physics and modeling. 2017;17(1):5-16 (in Russian).

13. Azarova OA. Complex conservative difference schemes for computing supersonic flows past simple aerodynamic forms. Comput Math and Math Phys. 2015;55(12):2025-2049.

14. Grassi T, Bovino S, Haugbølle, et al. A detailed framework to incorporate dust in hydrodynamical simulations. Monthly Notices of the Royal Astronomical Society. 2017;466(2):1259-1274.

15. Nishida $H$, Nonomura $T$, Abe $T$. Numerical study of plasma dynamics and electrohydrodynamic effect in DBD plasma actuator. International Journal of Plasma Environmental Science \& Technology. 2016;10(1):70-75. 